\title{
360-Degree Polarization Scanning with the Diversity of Boresight and Conical Patterns Switching
}

\author{
Roy B. V. B. Simorangkir ${ }^{1}$, Mengze $\mathrm{Li}^{2}$, Yang Yang ${ }^{3}, \mathrm{Kaida}_{\mathrm{Xu}}^{2}$, Karu Esselle${ }^{1}$, Eryk Dutkiewicz ${ }^{3}$ \\ ${ }^{1}$ Dept. of Engineering, Macquarie University, Sydney, NSW 2109, Australia, roy.basten@students.mq.edu.au \\ ${ }^{2}$ Institute of Electromagnetics and Acoustics \& Department of Electronic Science, Xiamen University, Xiamen 361005, China \\ ${ }^{3}$ School of Computing and Communications, University of Technology Sydney, Ultimo, NSW 2007, Australia, \\ yang.yang.au@ieee.org
}

\begin{abstract}
A novel reconfigurable antenna with the capability of 360-degree polarization scanning and the diversity of boresight and conical radiation patterns switching is presented in this paper. Each shorting-post is connected and controlled by a PIN diode to achieve the desired distribution of electric field null(s) in the substrate, where transverse magnetic (TM) modes switching between $T M_{11}$ and $T M_{02}$ can be accordingly realized corresponding to boresight and conical patterns. The antenna is analyzed in a cavity model, which, in terms of electric field, theoretically explains the generation of $T M_{11}$ and $T M_{02}$ resonant modes, and provides the audience with an innovative method of designing pattern reconfigurable antennas for the applications such as multiple-input and multiple-output (MIMO), wireless body sensor network (WBSN), Wi-Fi and WiMAX. The proposed antenna is with a competitive radius of $0.32 \lambda_{\mathrm{g}}$. The antenna structure is very easy to be fabricated. The measurement shows that the antenna can realize a boresight and conical pattern reconfiguration at $2.4 \mathrm{GHz}$ with a gain of $6 \mathrm{dBi}$ and efficiency of $86.7 \%$ (boresight mode), and $4.39 \mathrm{dBi}$ and efficiency of $82.4 \%$ (conical mode).
\end{abstract}

Index Terms - component, formatting, style, styling, insert.

\section{INTRODUCTION}

Pattern reconfiguration antennas are widely used in modern wireless communication systems with the advantages of dynamically switching radiation beams towards the desired directions [1]-[7]. Compared with smart antennas, pattern reconfiguration antennas can adaptively adjusting the electrical characteristics of the operating antennas in the varying cases to satisfy changing operating requirements. As the most interested radiation patterns in wireless communication applications, boresight and conical radiation patterns have been comprehensively studied in the applications of multiple-input and multiple-output (MIMO) systems [8][9], wireless body sensor network (WBSN) [10][11], Wi-Fi [3] and WiMAX [5]. The significance of realizing boresight and conical radiation patterns reconfiguration in a complex communication system can be vividly found in [6]-[13]. To the authors' best knowledge, it was rarely reported about the reconfiguration antennas in terms of boresight and conical radiation patterns switching until a L-probe patch antenna was proposed in [6], a U-slot antenna was presented in [7] and two square-ring patch antennas were reported in [12][13]. Over the past few years, these published works have shown considerable impact guiding antenna designs regarding radiation pattern diversity in MIMO system, WBSN and various complex multi-standard communication systems [2], [5], [12]. Theoretical analysis and design of planner antennas were well presented in [14] for boresight radiation pattern generation and [15] for conical radiation pattern generation. However, the theoretical analysis behind the boresight and conical pattern reconfiguration is still a gap. A well-presented analytical article like [16] will bring significant benefit for the audience who are seeking the true essence of this reconfiguration mechanism.

In this paper, we take the opportunity to demonstrate the previous work with more details which were not reported. As presented in [16], the antenna is based on a circular patch antenna with a set of concentrically shorted conductive vias, which are electronically controlled by PIN diodes. This kind of antenna can produce boresight or conical shaped radiation patterns by switching on the predefined groups of PIN diodes. The highlight of this work is that the reconfigurable boresight and conical radiation patterns are both designed at the same frequency band of $2.4 \mathrm{GHz}$. Therefore, this type of antenna can give more flexibility to design engineers when architecting complex communication systems such as MIMO, WBSN, WiFi and WiMAX. The circular shape of this antenna can appropriately meet most of the systems, which requires fast radiation switching between omnidirectional and directional performances.

\section{AntenNa Design}

\section{A. Overview}

Fig. 1 shows the structure of the proposed antenna, which is fabricated on a substrate of Rogers R04003C (thickness of $1.52 \mathrm{~mm}$ and $\varepsilon_{\mathrm{r}}=3.38$ ) with a mushroom-shaped conductor connected on top of the central feed point. The antenna is fed through a $50 \Omega$ coaxial transmission cable. The sideview, zoomed view and cut-sector view of the proposed antenna are presented in Fig. 1(b), (c) and (d), respectively. In this design, each shorting-post is controlled by a single PIN diode for the purpose of switching between ON and OFF status. The mushroom-shaped structure is applied for improvement of reflection coefficients particularly. Key performance of the proposed antenna is determined by the part of microstrip patch antenna. Therefore, the microstrip patch antenna will be analyzed in details in this article. Considering the gap width of 


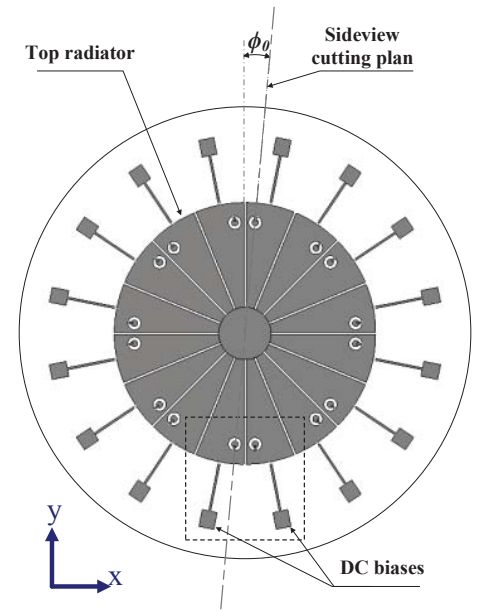

(a)

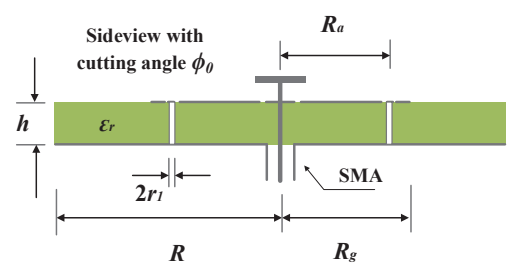

(b)

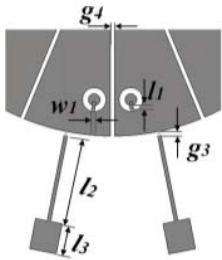

(c)

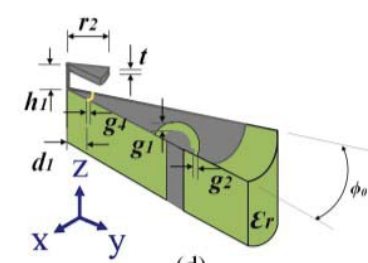

(d)
Fig. 1. Antenna configuration: (a) top view, (b) side view from cutting angle $\Phi_{0}$, (c) zoomed view of a pair of reconfigurable shorting posts, (d) a cut sector of the proposed antenna shown in (a), (dimentions given in [16]).

the ring and line slots is only $0.0016 \lambda_{0}$ which is much smaller than the wavelength $\lambda_{0}$, the impact from these slots on the resonance frequencies is relatively insignificant.

\section{B. Investigation on Number of Shorting Posts}

In [16], we presented the general idea of determination of the number of the shorting posts. However, it will be interesting to see a simultaneous variation of the number of shorting posts and the ones switched on. In Fig. 2, we take this opportunity to show the effect of varying $M$ to the electric field variation of the antenna in its $\mathrm{TM}_{11}$ mode, for a fixed value of $\mathrm{N}$. Note that $\mathrm{N}$ is the total number of the shorting post, while $\mathrm{M}$ is the number of the switched-on shorting post. As an example, we take the case where $\mathrm{N}=16$ and $\mathrm{M}$ is varying from 2 to 7 considering that it is not possible to achieve a symmetrical distribution of shorting post for the $\mathrm{TM}_{02}$ mode if only one shorting post that is switched on. It can be seen from Fig. 2 that the electric field null shifts to one side of the feed point when only 2 out of 16 shorting posts are switched on. As the number of $M$ increases, the electric field null can be moved very close to the feed point. Considering the desired field distribution and the minimum loss contributed

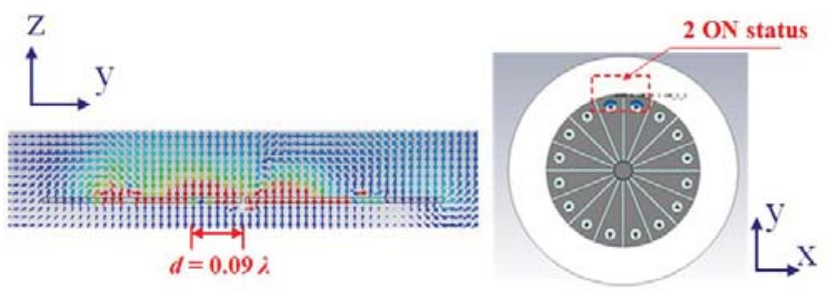

(a) $\mathrm{N}=16, \mathrm{M}=2$

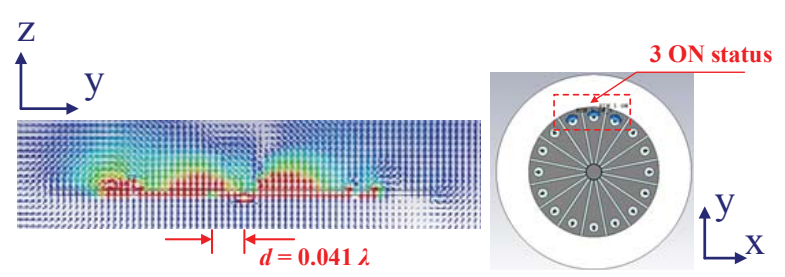

(b) $\mathrm{N}=16, \mathrm{M}=3$

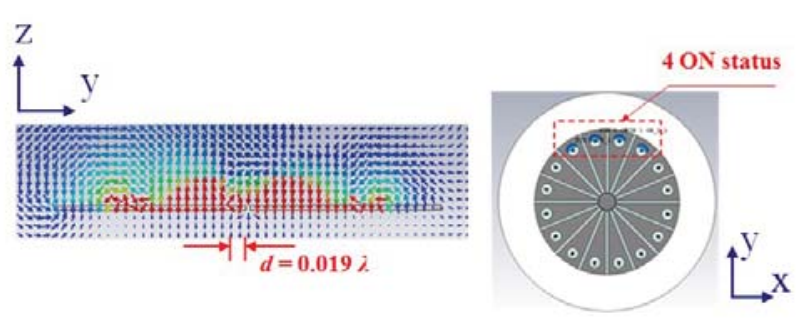

(c) $\mathrm{N}=16, \mathrm{M}=4$
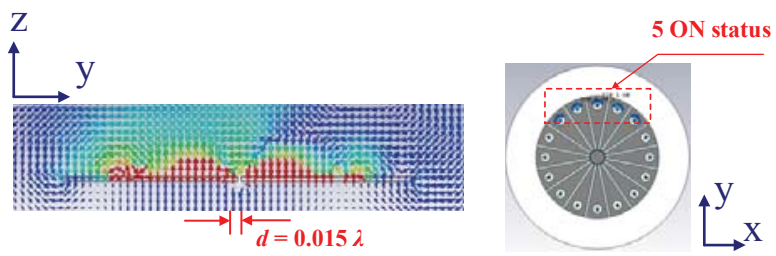

(d) $\mathrm{N}=16, \mathrm{M}=5$

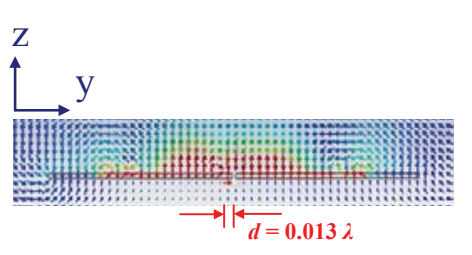

(e) $\mathrm{N}=16, \mathrm{M}=6$

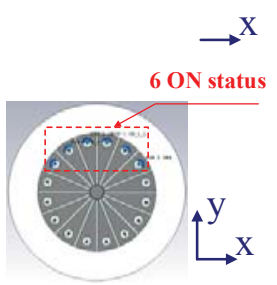

Fig. 2. Electric field variation with different number of shorting posts for a fixed $\mathrm{N}$ value. ( $\mathrm{N}$ is the total number of shorting posts, $\mathrm{M}$ is the number of the shorting posts which are switched on.)

by the diodes that are switched $\mathrm{ON}$, in this case, we switch on 4 shorting posts out of 16 shorting posts.

\section{360-DEGREE LINEAR-POLARIZATION SWITCHING}

The proposed antenna is capable of electronically switching linearly polarized waves with an angle of $22.5^{\circ}$, while propagating along $\mathrm{z}$-axis at $\mathrm{TM}_{11}$ mode (boresight pattern). If the 16 PIN diodes are clockwise-labeled from 1 to 16 and the PIN diodes 1-4 are switched on as Case 1 and PIN diodes 2-5 are switched on as Case 2, it can be clearly seen 


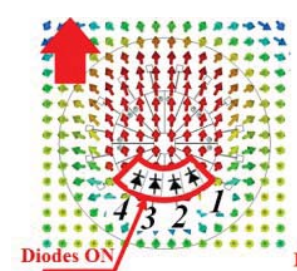

(a)

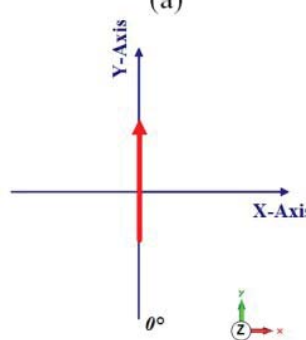

(c)

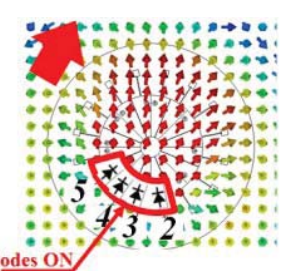

(b)

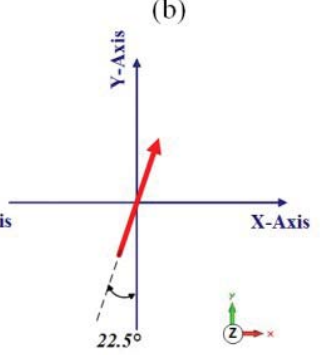

(d)
Fig. 3. Linear polarization of boresight mode at $2.4 \mathrm{GHz}$ : (a) electric field propagation in Case 1, (b) electric field propagation in Case 2, (c) vector direction in Case 1 and (d) vector direction in Case 2.

from Fig. 3(a) and (c) that the electric field vectors resonant along Y-axis, in contrast, these vectors are rotated $22.5^{\circ}$ around the electric field propagation direction $\mathrm{z}$-axis in Case 2 as is shown in Fig. 3(b) and (d). As the angle between the adjacent PIN diodes are $22.5^{\circ}$, the proposed antenna is capable of being switched to 16 different linear polarization status in terms of the concentrically distributed profile. This feature can be used as channel selection in point-to-point wireless communication system, such as MIMO, WBSN and Wi-Fi.

Beyond the advantage of steerable linear polarization angel, this antenna can also realize omnidirectional dual-beam steering in 3-D space. The dual-beam radiation patterns can be obtained by turning on opposite PIN diodes as is shown in Fig. 4, where three cases of switched-on PIN diodes combination are given. In Fig. 4(a) and (b), the gain patterns of Case 3 at the maximum beam directions are obtained on vertical and horizontal planes, respectively, here a dual-beam radiation pattern with peak gain of $3.7 \mathrm{dBi}$ and $3-\mathrm{dB}$ beamwidth of $99^{\circ}$ are observed at $2.4 \mathrm{GHz}$. The dual-beam radiation patterns do not significantly vary by increasing or decreasing the number of the switched-on opposite PIN diodes. As can be seen in Fig. 4(c), the resonant frequency can be shifted by $100 \mathrm{MHz}$ when PIN diodes are switched on as shown in Case 4 and Case 5.

\section{BORESIGHT AND CONICAL PATTERN RECONFIGURATION}

\section{A. Radiation Patterns}

The boresight and conical radiation patterns can be configured as: (a) boresight mode - turning on four adjacent switches and leave the rest switches off, see the top inset photo in Fig. 5, and (b) conical mode- turning on four switches evenly distributed at four directions around the antenna XOY plan and leave the rest switched off, see the bottom inset photo in Fig. 5. All the simulated results in this paper were obtained from Computer Simulation Technology (CST). The reflection

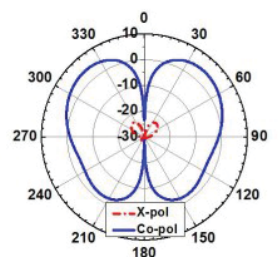

(a)

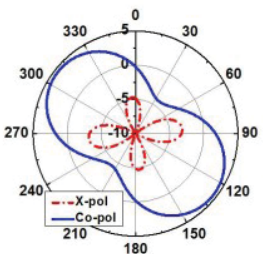

(b)
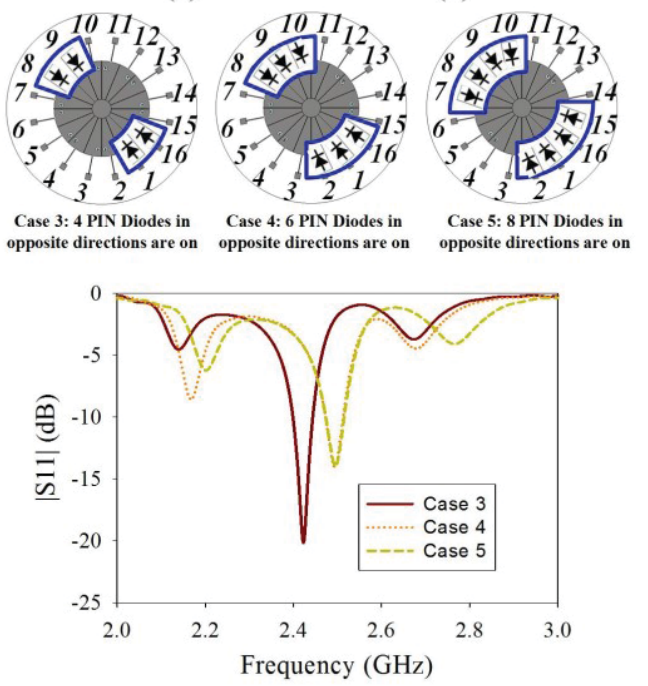

(c)

Fig. 4. Steerable dual-beam: (a) gain pattern in Case 3 obtained at $45^{\circ}$ from yz-plane, (b) gain pattern in Case 3 obtained at $48^{\circ}$ from xy-plane (c) reflection coefficients of Case 3 , Case 4 and Case 5 .

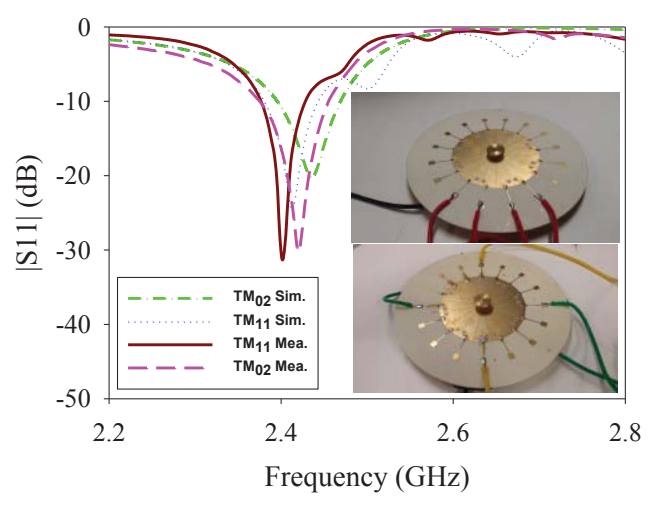

Fig. 5. Reflection coefficients for the microstrip monopolar patch antenna in $\mathrm{TM}_{11}$ mode and $\mathrm{TM}_{02}$ mode setup respectively.

coefficients and radiation patterns were measured using Keysight Vector Network Analyzer (VNA) and spherical nearfield (SNF) antenna measurement system, respectively. Fig. 5 shows that the simulated and measured reflection coefficients are with good agreement. It can also be observed that the measured resonant frequencies of $\mathrm{TM}_{11}$ and $\mathrm{TM}_{02}$ mode are well matched at $2.4 \mathrm{GHz}$. The measured resonant frequencies are slightly lower than the simulated ones due to the accuracy issues when fabricating. The measured bandwidth under $\mathrm{TM}_{11}$ mode is from $2.372 \mathrm{GHz}$ to $2.43 \mathrm{GHz}$ which is mostly 

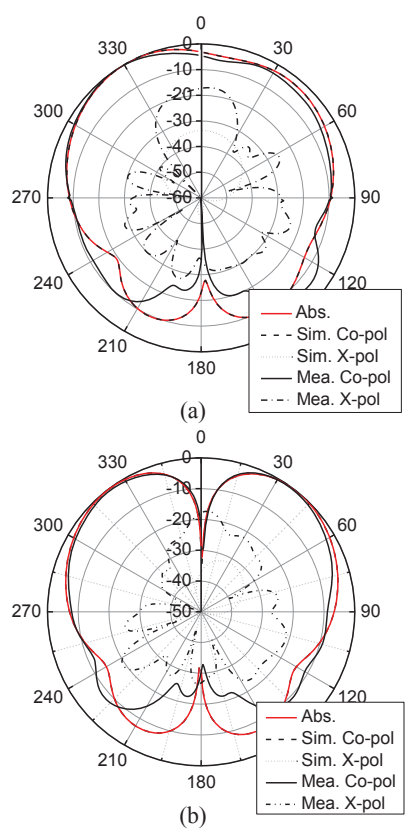

Fig. 6. Measured and simulated $z-y$ plane normalized radiation patterns of the proposed antenna at $2.4 \mathrm{GHz}$ (a) $\mathrm{TM}_{11}$ mode, (b) $\mathrm{TM}_{02}$ mode.

overlapped with the bandwidth of $2.375 \mathrm{GHz}-2.46 \mathrm{GHz}$ under $\mathrm{TM}_{02}$ mode.

Fig. 6 and 7 present the normalized z-y and z-x plane radiation patterns. It can be seen that the simulated and measured results are closely matched corresponding to each graph. Fig. 6 compares the radiation pattern switching results in $\mathrm{z}-\mathrm{y}$ plane between $\mathrm{TM}_{11}$ and $\mathrm{TM}_{02}$ modes. In the status of $\mathrm{TM}_{11}$ mode, a boresight radiation pattern is obtained with a maximum cross-polarization of $-18 \mathrm{~dB}$ in measurement. In contrast, the conical radiation pattern with the maximum power directed at elevation angle of $34^{\circ}$ is obtained with cross-polarization below $-18 \mathrm{~dB}$. In Fig. 7 (a), a boresight radiation pattern is obtained at far field with a conical shape co-plane polarization and boresight shape cross-plane polarization. This is caused by the weak electric fields generated by two electronic nulls at the far side of the circular substrate along x-axes. These two far located electronic field nulls are caused by the geometry size of the patch according to (1) and (2). However, the impact of these two nulls on $\mathrm{TM}_{11}$ mode is limited owing to that an electronic field null at the center of the substrate is able to generate a dominating $\mathrm{TM}_{11}$ electronic field, of which the directions are opposite in the substrate along y-axes. Fig. 7 (b) presents $\mathrm{TM}_{02}$ mode $\mathrm{z}$ - $\mathrm{x}$ plane radiation patterns, of which a conical co-plane radiation pattern with a maximum power elevation angle of $33^{\circ}$ is observed. The cross-plane radiation power level is more than $20 \mathrm{~dB}$ lower than its corresponding co-plane radiation pattern.

\section{B. Antenna Gain}

The simulated and measured gain under $\mathrm{TM}_{11}$ and $\mathrm{TM}_{02}$ modes are shown in Fig. 8, respectively. It can be seen that the measured gain under each mode is well matched to its

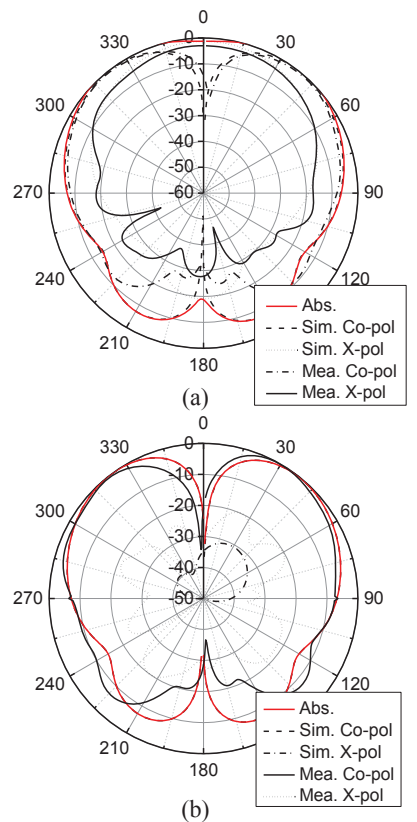

Fig. 7. Measured and simulated z-x plane normalized radiation patterns of the proposed antenna at $2.4 \mathrm{GHz}$ (a) TM11 mode, (b) TM02 mode.

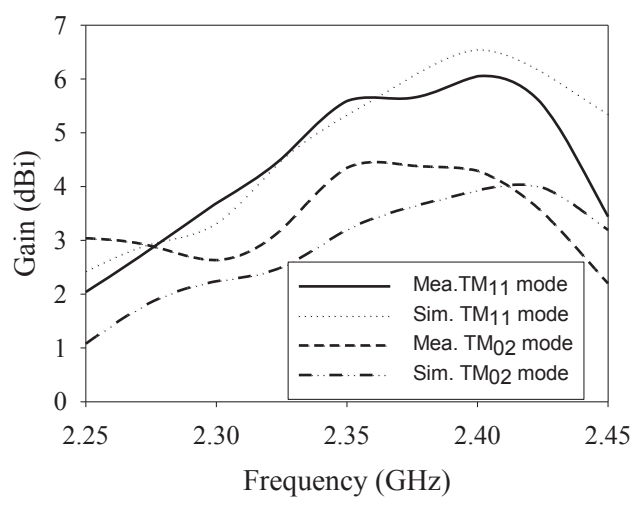

Fig. 8. Reflection coefficients for the microstrip monopolar patch antenna.

corresponding simulated gain. The measured peak gain in $\mathrm{TM}_{11}$ mode is $6 \mathrm{dBi}$ at $2.4 \mathrm{GHz}$, while the measured peak gain of $\mathrm{TM}_{02}$ mode is $4.39 \mathrm{dBi}$ at $2.38 \mathrm{GHz}$. Since the narrow slots are applied on the antenna patch, the loss caused by the PIN diodes can be effectively reduced. Consequently, we obtained a relatively high measured antenna efficiency of $86.7 \%$ for $\mathrm{TM}_{11}$ mode and $82.4 \%$ for $\mathrm{TM}_{02}$ mode at $2.4 \mathrm{GHz}$, where the resonant frequencies of the two modes are overlapped. While not shown in parameter study, it is found that the realized antenna gain can be slightly increased if the adjacent shortingposts are placed towards each other. While $\Phi_{0}=6^{\circ}$ as shown in Fig. 1(a), the antenna gain can be increased by $0.8 \mathrm{dBi}$ compared with the case that shorting-posts are placed at $\Phi_{0}=11.25^{\circ}$ (symmetric center of a sector).

\section{Antenna Efficiency}

The antenna efficiency using ideal switches and real models of MA4FCP300 is studied. The comparisons for both 


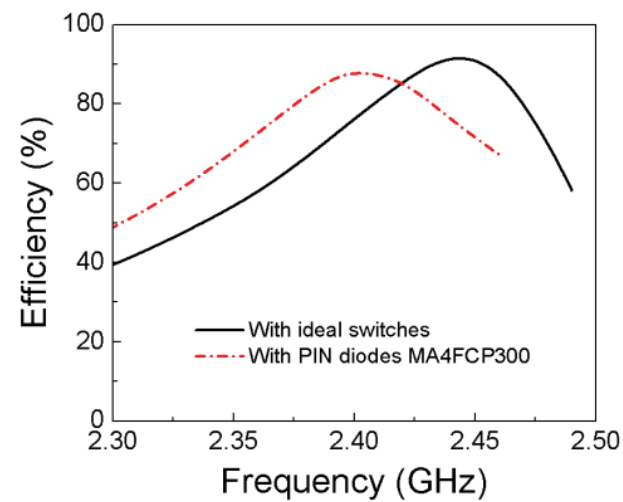

(a)

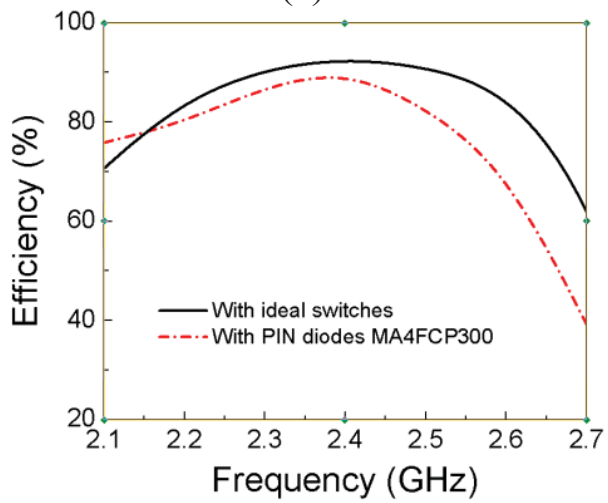

(b)

Fig. 9 Efficiency study of the proposed antenna using PIN diode MA4FCP300 and ideal switches: (a) operating at $\mathrm{TM}_{11}$ mode, and (b) operating at $\mathrm{TM}_{02}$ mode

cases, $\mathrm{TM}_{11}$ and $\mathrm{TM}_{02}$ modes, are given in Fig. 9. As can be seen, the antenna efficiencies with the real model are only around $3 \%$ and $8 \%$ less than the one with ideal switches for $\mathrm{TM}_{11}$ and $\mathrm{TM}_{02}$ modes, respectively. This can be understood because the ohmic resistance of the selected PIN diodes is considerably low $(2.6 \mathrm{Ohm})$. Therefore, when the RF signal goes through the pin diodes that are switched on, the ohmic losses $\left(\mathrm{I}^{2} \mathrm{R}\right)$ contributed by the diodes is low. On the other hand, for those diodes that are switched off, the RF currents that leak through, due to the nature of the non-ideal switch, are very low. Thus, the loss contributed by them can be considered negligible.

\section{CONCLUSION}

This paper presented some extended work which is not fully demonstrated in previous work [16]. As a novel design, the presented work has the pattern reconfigurablility switching between the boresight and conical modes. 16 shorting-posts, each of which can be switched on and off through a PIN diode. Taking the advantage of this feature, we have successfully realize a reconfigurable antenna with 360-degree linearpolarization switching as well as the diversity of boresight and conical radiation patterns.

\section{REFERENCES}

[1] S. L. S. Yang and K. M. Luk, "A wideband L-probes fed circularlypolarized reconfigurable microstrip patch antenna," IEEE Trans. Antennas Propag., vol. 56, no. 2, pp. 581-584, Feb. 2008.

[2] P. K. Li, Z. H. Shao, Q. Wang and Y. J. Cheng, "Frequency- and pattern-reconfigurable antenna for multistandard wireless applications," IEEE Antennas Wireless Propag. Lett., vol. 14, pp. 333-336, 2015.

[3] B. Majumdar, and K. P. Esselle, "A dual-mode reconfigurable patch antenna and an extended transmission line model," Microw. Opt. Tech. Lett., vol. 58, no. 1, pp. 57-61, Jan. 2016.

[4] M. Jusoh, T. Aboufoul, T. Sabapathy, A. Alomainy and M. R. Kamarudin, "Pattern-Reconfigurable Microstrip Patch Antenna with Multidirectional Beam for WiMAX Application," IEEE Antennas Wireless Propag. Lett., vol. 13, pp. 860-863, 2014.

[5] T. Aboufoul, C. Parini, X. Chen and A. Alomainy, "Patternreconfigurable planar circular ultra-wideband monopole antenna," IEEE Trans. Antennas Propag., vol. 61, no. 10, pp. 4973-4980, Oct. 2013.

[6] S. L. S. Yang and K. M. Luk, "Design of a wide-band L-probe patch antenna for pattern reconfiguration or diversity applications," IEEE Trans. Antennas Propag., vol. 54, no. 2, pp. 433-438, Feb. 2006.

[7] P. Y. Qin, Y. J. Guo, A. R. Weily and C. H. Liang, "A pattern reconfigurable U-slot antenna and its applications in MIMO systems,' IEEE Trans. Antennas Propag., vol. 60, no. 2, pp. 516-528, Feb. 2012.

[8] D. Piazza, N. J. Kirsch, A. Forenza, R. W. Heath and K. R. Dandekar, "Design and evaluation of a reconfigurable antenna array for MIMO systems," IEEE Trans. Antennas Propag., vol. 56, no. 3, pp. 869-881, Mar. 2008.

[9] Y. Zhou, R. S. Adve and S. V. Hum, "Design and evaluation of pattern reconfigurable antennas for MIMO applications," IEEE Trans. Antennas Propag., vol. 62, no. 3, pp. 1084-1092, Mar. 2014.

[10] J. J. Tak, S. Lee and J. Choi, "All-textile higher order mode circular patch antenna for on-body to on-body communications," IET Micro. Antennas Propag., vol. 9, no. 6, pp. 576-584, Apr. 2015.

[11] R. B. V. B. Simorangkir, Y. Yang, L. Matekovits, K. P. Esselle, ”Dualband dual-mode textile antenna on PDMS substrate for body-centric communications," IEEE Antennas and Wirel. Propag. Lett., vol. 16, pp. 677-680, 2017.

[12] S. H. Chen, J. S. Row, and K. L. Wong, "Reconfigurable square-ring patch antenna with pattern diversity," IEEE Trans. Antennas Propag., vol. 55 , no. 2 , pp. 472-475, Feb. 2007.

[13] W. L. Liu, T. R. Chen, S. H. Chen, and J. S. Row, "Reconfigurable microstrip antenna with pattern and polarization diversities," Electron. Lett., vol. 43, no. 2, pp. 77-78, Jan. 2007.

[14] J. Liu, Q. Xue, H. Wong, H. W. Lai, and Y. Long, "Design and analysis of a low-profile and broadband microstrip monopolar patch antenna," IEEE Trans. Antennas Propag., vol. 61, no. 1, pp. 11-18, Jan. 2013.

[15] L. Ge, and K. M. Luk, "Frequency-reconfigurable low-profile circular monopolar patch antenna," IEEE Trans. Antennas Propag., vol. 62, no. 7, pp. 3443-3449, Jul. 2014.

[16] Y. Yang, R. B. V. B. Simorangkir, X. Zhu, K. P. Esselle and Q. Xue, "A novel boresight and conical pattern reconfigurable antenna with the diversity of 360-degree polarization scanning," IEEE Trans. Antennas Propag., early access, accepted in Sep 2017. 